\title{
Mycological Profile in Otomycosis Patients: A Cross Sectional Hospital Based Study in Tertiary Care Centre
}

\author{
Nitin Ankale, ${ }^{1}$ Jyoti M Nagmoti, ${ }^{1}$ Utkarsh Anand ${ }^{1}$
}

\section{Introduction}

\section{ABSTRACT}

This study was conducted to study the fungal profile in otomycosis patients in the tropical region of North Karnataka. A total of 108 samples of symptomatic otomycosis were investigated in this study.

Materials and Methods

Aural swabs were collected on 1st visit and these swabs were immediately inoculated over Sabouraud's dextrose agar media and incubated at 37 degree Celsius for culture of fungi.

$\underline{\text { Results }}$

Fungal pathogens were isolated in 89 samples, 18 samples were negative and 1 sample was reported as being contaminated. Fungi belonging to genus Aspergillus was isolated in $92.11 \%$ of cases of which Aspergillus niger was commonest isolated in $38(41.57 \%)$ followed br Aspergillus flavus 32(35.95\%) and Aspergillus fumigatus 7(7.86\%). Candida species were found in 2(2.24\%) and Mucor in 1(1.12\%). The most common symptom was Itching 91(84.25\%). In this study ear discharge was the commonest finding (44.44\%) followed by Black mycotic plug (28.70\%).

\section{Conclusion}

Otomycosis is a condition encountered in hot, humid climate with symptoms like itching and ear discharge. Aspergillus and Candida are the fungal species responsible for majority of cases. Local antifungal treatment with measures like keeping the ear dry resolves most of the cases.

Keywords

Otomycosis; Aspergillus; Candida

$\mathrm{O}$ tomycosis or fungal otitis externa has typically been described as fungal infection of the external auditory canal with infrequent complications involving the middle ear. ${ }^{1}$ Otomycosis is one of the common conditions encountered in a general otolaryngology clinic. Symptoms usually include itching, otalgia, otorrhoea, ear blockage, hearing loss and tinnitus. ${ }^{2,3,4}$ It is worldwide in distribution with more prevalence in warm, wet, humid and dusty environment of the tropics and subtropics. ${ }^{1,5,7,9}$ Its incidence has been increasing due to the increased use of antibiotics, immunocompromised host conditions such as diabetes and more recently increased use of topical antibioticssteroid combination and bad aural hygiene (instilling oil and water in the ear). ${ }^{1,10,12}$

Other factors that predispose patients to otomycosis include: open mastoid cavities, hearing aids with occlusive moulds, trauma and bacterial infection. ${ }^{11,12}$ Although rarely life threatening, in immuno-compromised patients otomycosis can lead to skull base osteomyelitis along with multiple cranial nerve palsies with serious mortality and morbidity. ${ }^{10,11,13,14,15}$ The disease is challenging and frustrating for both patients and otolaryngologists as it frequently requires long term treatment and follow up. This study was devised to review the fungal profile in otomycosis patients in the tropical region of North Karnataka.

1 - JNMC, Nehru nagar, Belagavi
Corresponding author:
$\begin{aligned} & \text { Dr Utkarsh Anand } \\ & \text { email: utkarshanand117@gmail.com }\end{aligned}$

email: utkarshanand117@gmail.com 
Table I: Symptoms with which the patient presented

\begin{tabular}{|c|c|c|}
\hline COMPLAINTS & TOTAL NO OF AFFECTED EARS & PERCENTAGE \\
\hline Itching in the Ear & 91 & 84.25 \\
\hline Otalgia & 77 & 71.29 \\
\hline Decreased Hearing & 70 & 64.81 \\
\hline Ear Discharge & 50 & 46.29 \\
\hline Tinnitus & 9 & 8.33 \\
\hline
\end{tabular}

\section{Materials and Methods}

This cross sectional study was done in a tertiary care centre in the tropical region of North Karnataka, with the objective to study the fungal profile in clinically diagnosed patients of otomycosis in ENT outpatient as well as inpatient departments.

The samples were procured from the ear using sterile cotton swab on the 1st visit and immediately transported to the microbiology laboratory. The swabs were immediately inoculated over Sabouraud's dextrose agar media and incubated at 37 degree Celsius. A total of 108 samples of symptomatic otomycosis were investigated in this study.

The debris in the external auditory canal was thoroughly cleaned by suction aspiration and dry mopping and patients were put on antifungal ear drops.

\section{Results}

In our study males $(54.62 \%)$ were more than females $(45.38 \%)$. Youngest patient was 2 years old while the oldest was an 86 years old male. Occupationally most of the patients were farmers $(34.25 \%)$ followed by students (15.74\%), businessmen (15.74\%), and housewives (13.8\%). Other groups included drivers, service personnel and teachers. Left ear was more commonly involved $(49.07 \%)$ while incidence on right side was $34.86 \%$. Most common symptom at presentation was ear itching (84.95\%). (Table I)
Most of the samples i.e. $62.94 \%$ were collected between June to September when the incidence of otomycosis increased, probably due to the rainy season.

On otoscopic examination $45 \%$ of the ears had discharge and COM, 28.7\% had black mycotic plug, $9.25 \%$ had wet mycelial mat, $7.4 \%$ had dry mycelial mat, $5.55 \%$ had soft debris and $4.62 \%$ had cotton woolly mat.

We did a mycological profile of the samples collected and on growing over Sabouraud's dextrose agar the most commonly isolated group was Aspergillus. Among the Aspergillus group the most common species isolated

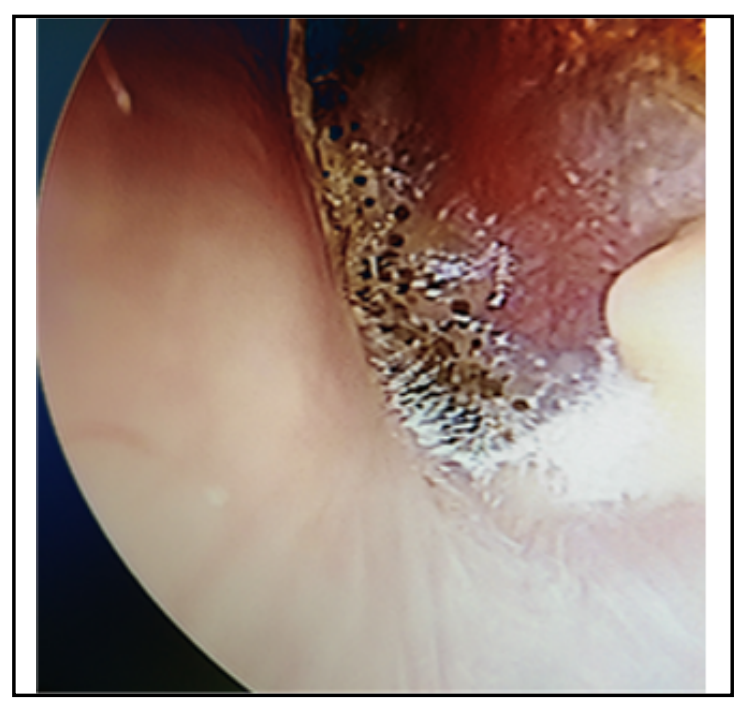

Fig.1. Clinical photograph of left external auditory canal showing black otomycotic debris suggestive of aspergillus niger (Otoscopic view) 
Table II: The fungal profile

\begin{tabular}{|c|c|c|}
\hline FUNGAL SPECIES & TOTAL NO OF SAMPLES & PERCENTAGE \\
\hline Aspergillus niger & 38 & 41.57 \\
\hline Aspergillus flavus & 32 & 35.95 \\
\hline Aspergillus fumigatus & 7 & 7.86 \\
\hline Aspergillus versicolor & 3 & 3.37 \\
\hline Aspergillus tertius & 2 & 2.24 \\
\hline Aspergillus glacus & 1 & 1.12 \\
\hline Candida glabrata & 1 & 1.12 \\
\hline Candida lipolytica & 1 & 1.12 \\
\hline Verticillium species & 1 & 1.12 \\
\hline Rhizopus species & 1 & 1.12 \\
\hline Alternaria species & 1 & 1.12 \\
\hline Mucor species & 1 & 1.12 \\
\hline
\end{tabular}

was Aspergillus niger (41.57\%), followed by Aspergillus flavus (35.95\%) and Aspergillus fumigatus (7.86\%). (Fig.1) Other species isolated were Candida species, Rhizopus species, Verticillium species, Alternaria species and Mucor species. (Table II)

Patients were treated with Clotrimazole ear drops for 7 days. In cases with partial/no response, Fluconazole was added both orally and topically( in the form of ear drops) for 2 weeks. All the patients responded well.

\section{Discussion}

Otomycosis is a chronic infective disorder of the ear and remains a frustrating condition for the clinicians and is a source of misery for the infected patients. The term is used to denote fungal infection of the external auditory canal. The incidence of this condition is not known but it has been found that out of 8 cases of otitis externa, 1 is of fungal origin. Of the cases diagnosed with otomycosis, $90 \%$ are caused by Aspergillus and the rest by Candida species. ${ }^{16}$ Incidence of otomycosis is higher in hot climates and much of the literature has originated from the tropical and subtropical regions of the world. An American study has found that the incidence of otomycosis peaked during the summer months. Various factors have been held responsible for otitis externa in general and otomycosis in particular. These include high humidity, increased temperature and local trauma usually from the use of cotton swabs.

Cerumen which is present in the external auditory canal has a $\mathrm{pH}$ of 4 to 5 and hence it supresses the bacterial and fungal growth. Aquatic sports including swimming and surfing are particularly associated with this condition because repeated exposure to water removes the cerumen from the external auditory canal and results in the drying of external auditory canal. ${ }^{17}$ 
The mycological infection results in masses of debris containing hyphae and suppuration. Pruritus is a marked feature of this condition and discharge is often present. ${ }^{10,18}$

The initial presentation is similar to bacterial infection but the fungal infection of the ear is characterised by many long, white, filamentous hyphae which are growing from the skin of the external auditory canal. Suspicion of otomycosis only arises when the condition fails to resolve with standard antibiotic treatment. ${ }^{19}$

In order to treat otomycosis, the external auditory canal should be cleaned of the debris and discharge as these lower the $\mathrm{pH}$ of the external auditory canal and hence reduce the activity of aminoglycoside ear drops (used in those patients who showed signs and symptoms suspicious of bacterial otitis externa). Suction may be done for this. Ear should be kept dry and scratching of the ear with cotton bud should be avoided. Antifungal ear drops are of value in treating such patients. ${ }^{20}$

\section{Conclusion}

Otomycosis is a common condition encountered in this geographical area where the humid weather is favourable for the growth of fungi. The disease was found to be predominantly unilateral (84.25\%) and only $15.75 \%$ of cases were bilateral. Otomycosis shows a seasonal preponderance with $62.94 \%$ of cases in this study presenting between June to September which is the rainy season. Ear itching was the most common symptom $(84.25 \%)$ at presentation. Commonest sign was ear discharge, seen in $44.44 \%$ of cases. The condition was commonly found in people working in dusty environment particularly farmers, businessmen, students who are exposed to the outdoor environment and housewives who work in a cold damp environment. In this study Aspergillus group were found to be the most common causative agent. Of the Aspergillus group the common isolated species were Aspergillus niger (41.57\%) followed by Aspergillus flavus (35.95\%), Aspergillus fumigatus (7.86\%), Aspergillus versicolor (3.37\%), Aspergillus tertius (2.24\%) and Aspergillus glacus $(1.12 \%)$. Other species isolated included Candida glabrata.
Topical antifungal medications along with measures like keeping the ears dry will resolve most of the cases of Otomycosis, while in some cases it might be required to be supplemented by oral antifungal medication.

\section{References}

1. Haja AN, Shaik KM, Siva Subba RP. Mycology of otomycosis in a tertiary care teaching hospital. J Res Med Den Sci. 2015 Jan-Mar; 3(1):27-30

2. Pradhan B, Tuladhar N, Amatya R. Prevalence of otomycosis in outpatient department of otolaryngology in tribhuvan university teaching hospital, kathmandu, nepal. Ann Otol Rhinol Laryngol. 2003 Apr; 112(4):384-7

3. Ozcan K M, Ozcan M, Karaarslan A et al. Otomycosis in turkey: predisposing factors, aetiology and therapy. J Laryngol Otol. 2003 Jan; 117(1):39-42

4. Chandra J, Maini S, Subrahmanyan S et al. Otomycosis- a clinic-mycological study and efficacy of mercurochrome in its treatment. Mycopathologia 1996 Feb; 135(1):9-12

5. Mgbe R, Umana A, Adekanye A et al. Otomycosis - a management challenge in calabar, south-south nigeria. Int $\mathrm{J}$ Third World Med. Jan 2010; 9(2):1

6. Chapparbandi R B, Kazi F N, Ali K. Otomycosis: an overview in hyderabad karnataka region. J Evolu Med Dental Sci. 2014 Sep; 3(46):11213-16

7. Fasunla J, Ibekwe T, Onakoya P. Otomycosis in western Nigeria. Mycoses. 2008 Jan; 51(1):67-70

8. Munguia R, Daniel S J. Ototopical antifungals and otomycosis: a review. Int J Paediatr Otorhinolarygol. 2008 Apr; 72(4):453-9

9. Pontes Z B, Silva A D, Lima Ede O, Guerra Mde H. Otomycosis a retrospective study. Braz J Otorhinolaryngol. 2009 May-Jun; 75(3):367-70

10. Satish H S, Vishwanatha B, Manjuladevi M. A clinical study of otomycosis, IOSR J Dental Med Sci. 2013 Mar-Apr;5(2):57-62

11. Mahmoudabadi A Z, Masoomi S A, Mohammadi H. Clinical and mycological studies of otomycosis. Pak J Med Sci. 2010Jan-Mar; 26(1):187-90

12. Anwar K, Muhammad S G. Otomycosis; clinical features, predisposing factors and treatment implications, Pak J Med Sci. 2014 May-Jun; 30(3): 564-7

13. Mugliston T, O'Donoghue G. Otomycosis a continuing problem. J Laryngol Otol. 1985 Apr; 99(4): 327-33

14. Yehia M M, Al-Habib H M, Shehab N M. Otomycosis: a common problem in north Iraq, J Laryngol Otol. 1990 May; 104(5):387-9

15. Yadav S P S, Gulia J S, Jagat S, Aggrawal N. Role of ototopical fluconazole and clotrimazole in management of otomycosis. Ind J Otol. 2007 Dec; 13:12-5 
16. Frank A M, Smit W, Nicolaas P A, et al. Clinical efficacy of three common treatments in acute otitis externa in primary care: randomised controlled trial. 2003 Nov;327(7425):1201-05

17. Kujundzic M, Braut T, Manestar D, et al. Water related otitis externa; Coll Antropol. 2012 Sep; 36(3):893-7

18. Ho T, Vrabec J T, Yoo D, et al. Otomycosis: clinical features and treatment implications; Otolaryngol Head Neck Surg. 2006 Nov; 135(5):787-91
19. Abou-Halawa A S, Khan M A, Alrobaee A A, et al. Otomycosis with perforated tympanic membrane: self medication with topical antifungal solution vs medicated ear wick; Int $\mathrm{J}$ Health Sci. 2012 Jan; 6(1):73-7

20. Pabla L, Jindal M, Latif $\mathrm{K}$. The management of otitis externa in UK general practice. Eur Arch Otorhinolaryngol. 2012 Mar; 269(3):753-6. 